\title{
LARGE ABUNDANCES OF POLYCYCLIC AROMATIC HYDROCARBONS IN TITAN'S UPPER ATMOSPHERE
}

\author{
M. López-Puertas ${ }^{1}$, B. M. Dinelli ${ }^{2}$, A. Adriani ${ }^{3}$, B. Funke $^{1}$, M. García-Comas ${ }^{1}$, \\ M. L. Moriconi ${ }^{4}$, E. D'Aversa ${ }^{3}$, C. Boersma ${ }^{5}$, And L. J. Allamandola ${ }^{5}$ \\ ${ }^{1}$ Instituto de Astrofísica de Andalucía (CSIC), E-18080 Granada, Spain; puertas@iaa.es \\ 2 ISAC-CNR, I-40129 Bologna, Italy \\ ${ }^{3}$ IAPS-INAF, I-00133 Rome, Italy \\ ${ }^{4}$ ISAC-CNR, I-00133 Rome, Italy \\ 5 NASA Ames Research Center, Moffett Field, CA 94035-1000, USA \\ Received 2013 February 28; accepted 2013 April 4; published 2013 June 5
}

\begin{abstract}
In this paper, we analyze the strong unidentified emission near $3.28 \mu \mathrm{m}$ in Titan's upper daytime atmosphere recently discovered by Dinelli et al. We have studied it by using the NASA Ames PAH IR Spectroscopic Database. The polycyclic aromatic hydrocarbons (PAHs), after absorbing UV solar radiation, are able to emit strongly near $3.3 \mu \mathrm{m}$. By using current models for the redistribution of the absorbed UV energy, we have explained the observed spectral feature and have derived the vertical distribution of PAH abundances in Titan's upper atmosphere. PAHs have been found to be present in large concentrations, about $(2-3) \times 10^{4}$ particles $\mathrm{cm}^{-3}$. The identified PAHs have 9-96 carbons, with a concentration-weighted average of 34 carbons. The mean mass is $\sim 430 \mathrm{u}$; the mean area is about $0.53 \mathrm{~nm}^{2}$; they are formed by 10-11 rings on average, and about one-third of them contain nitrogen atoms. Recently, benzene together with light aromatic species as well as small concentrations of heavy positive and negative ions have been detected in Titan's upper atmosphere. We suggest that the large concentrations of PAHs found here are the neutral counterpart of those positive and negative ions, which hence supports the theory that the origin of Titan main haze layer is located in the upper atmosphere.
\end{abstract}

Key words: molecular processes - planets and satellites: atmospheres - planets and satellites: composition - planets and satellites: individual (Titan) - radiation mechanisms: non-thermal

Online-only material: color figures

\section{INTRODUCTION}

The idea of Titan's lower atmosphere thick haze layer being generated from complex organic molecules has been proposed for a long time (Smith et al. 1981; Sagan et al. 1984). Major advances were achieved in the 1980s with models predicting the formation of heavy species (propane, butane, polyacetylenes, and cyanoacetylenes) from the simpler nitrogen and methane molecules (Yung et al. 1984). Laboratory simulations have also shown that the formation of polycyclic aromatic hydrocarbons (PAHs) is feasible in Titan's atmospheric conditions (Sagan et al. 1993). The detection of benzene in Titan's stratosphere by the Infrared Space Observatory (Coustenis et al. 2003) represented a major step forward. This discovery and the preparation for the Cassini mission triggered model studies for explaining benzene formation. As a result, these models subsequently predicted that PAHs could be the largest contributors of Titan's haze (Wilson \& Atreya 2003; Lebonnois et al. 2002; Wilson et al. 2003). For many years it was assumed that the major haze layer was mainly generated where it resides, about a few hundred kilometers above the surface (Wilson \& Atreya 2003; Lebonnois et al. 2002). However, recent detection of benzene and other light aromatic species in the thermosphere $(\sim 1000 \mathrm{~km})$ by the Ion and Neutral Mass Spectrometer (INMS) on board Cassini (Waite et al. 2007, 2010), and measurements of significant concentrations (several hundred particles $\mathrm{cm}^{-3}$ ) of heavy positive and negative ions, with masses between 1000 and 10,000 u, in Titan's ionosphere by the Cassini Plasma and Electron Spectrometer (CAPS/ELS; Coates et al. 2007, 2010;
Crary et al. 2009; Waite et al. 2010) hint at the presence of high concentrations of complex carbon-nitrogen neutrals and suggest that haze aerosols may form higher up in the atmosphere and descend to the lower regions (Waite et al. 2007; Vuitton et al. 2008; Krasnopolsky 2009; Lavvas et al. 2011). This hypothesis implies the generation of large amounts of heavy aromatic species at high altitudes (Waite et al. 2007; Vuitton et al. 2008; Lavvas et al. 2011; Ricketts et al. 2008). However, to date, they have not yet been experimentally confirmed; nor have their identity, composition, abundance, and distribution been revealed (Waite et al. 2007, 2010; Atreya 2007).

The Visual-Infrared Mapping Spectrometer (VIMS) has probed Titan's thermosphere during many flybys. VIMS limb daytime observations in the $3.0 \mu \mathrm{m}$ and $3.3 \mu \mathrm{m}$ regions have been recently analyzed to derive $\mathrm{HCN}$ and $\mathrm{CH}_{4}$ density profiles in Titan's upper atmosphere (500-1100 km; Adriani et al. 2011; García-Comas et al. 2011). In a recent paper, Dinelli et al. (2013) have reported the finding of an unidentified emission near $3.28 \mu \mathrm{m}$ in Titan's upper daytime atmosphere measured by the Cassini/VIMS instrument. They suggested that its spectral signature resembles that of aromatic compounds, e.g., PAHs and/or heterocyclic aromatic compounds (HACs). However, the question remains as to how, being in much smaller concentrations than $\mathrm{CH}_{4}$ (Waite et al. 2007; Vuitton et al. 2008), they can emit an emission comparable to that of the strong $3.3 \mu \mathrm{m}$ bands of the abundant $\mathrm{CH}_{4}$. In this paper, we analyze this emission in detail by using the NASA Ames PAH IR Spectroscopic Database (Bauschlicher et al. 2010) and the AmesPAHdbIDLSuite set of IDL tools (Boersma et al. 2010, 
Table 1

VIMS Observations Used in This Study

\begin{tabular}{lcccrr}
\hline \hline Encounter/Sequence & Cube & $\begin{array}{c}\text { Approximated IFOV } \\
(\mathrm{km})\end{array}$ & Phase Angle & No. of Profiles & Geolocations \\
\hline T34/S32-GLOBMAP003 & 1563524168 & 50 & $60^{\circ}$ & 5 & $60^{\circ} \mathrm{SE}, 65^{\circ} \mathrm{SE}, 70^{\circ} \mathrm{SE}, 75^{\circ} \mathrm{SE}, 80^{\circ} \mathrm{SE}$ \\
T34/S32-GLOBMAP003 & 1563524658 & 47 & $60^{\circ}$ & 6 & $55^{\circ} \mathrm{SE}, 60^{\circ} \mathrm{SE}, 65^{\circ} \mathrm{SE}, 70^{\circ} \mathrm{SE}, 75^{\circ} \mathrm{SE}, 80^{\circ} \mathrm{SE}$ \\
T34/S32-GLOBMAP003 & 1563525149 & 45 & $60^{\circ}$ & 6 & $45^{\circ} \mathrm{SE}, 50^{\circ} \mathrm{SE}, 55^{\circ} \mathrm{SE}, 60^{\circ} \mathrm{SE}, 65^{\circ} \mathrm{SE}, 70^{\circ} \mathrm{SE}$ \\
T35/S33-CIRSSTARE001 & 1567269485 & 70 & $26^{\circ}$ & 3 & $60^{\circ} \mathrm{SW}, 40^{\circ} \mathrm{SW}, 0^{\circ} \mathrm{W}$
\end{tabular}

Note. Integration time was $640 \mathrm{~ms}$ in all cases.

2011), appropriately modified. In addition, we discuss whether the concentration of the PAHs (or HACs) that we find can shed some light on their role on the haze formation.

\section{THE UNIDENTIFIED $3.28 \mu \mathrm{m}$ EMISSION}

Dinelli et al. (2013) recently reported an unidentified emission near $3.28 \mu \mathrm{m}$ in Titan's upper daytime atmosphere measured by the VIMS instrument on board Cassini. Although it was derived from only two flybys, it is a persistent feature present in all daytime measurements taken so far by VIMS. In this study, we analyze the data taken during two Titan flybys: T34 (2007 July 19) and T35 (2007 August 31). The major characteristics of the data are summarized in Table 1.

Briefly, VIMS is an imaging spectrometer that takes images with a resolution of $64 \times 64$ pixels at 352 separate wavelengths (spectral cubes). The infrared channel $(0.85-5.2 \mu \mathrm{m})$ analyzed here has a nominal angular resolution of $0.5 \times 0.5 \mathrm{mrad}$ and a spectral resolution of $16 \mathrm{~nm}$ (Brown et al. 2004). During the flybys to Titan, VIMS is capable of measuring Titan's upper atmospheric limb emission from its surface up to about $1500 \mathrm{~km}$ with a moderate vertical resolution $(22-70 \mathrm{~km})$. Among the acquired measurements taken during the two flybys we have selected the spectral cubes that cover the highest altitudes (above 400-500 km). Also, in order to minimize potential stray-light contaminations and to have the largest atmospheric signal, we have used the measurements with the largest integration time (640 ms), with the best vertical resolution, and with a phase angle lower than $60^{\circ}$. A total of 20 sequences, all of them in the southern hemisphere, were used (see Table 1). From the selected cubes, we extracted sequences of spectra sampling the limb of Titan's atmosphere at different tangent heights along lines perpendicular to Titan's surface (see Figure 1 in GarcíaComas et al. 2011). The spectral and radiometric calibration of the data was carried out using the latest VIMS pipeline (RC17). The instrument noise error near $3.3 \mu \mathrm{m}$ is about $30 \mathrm{nW} \mathrm{m} \mathrm{mm}^{-1} \mathrm{sr}^{-1}$. More details on the observations and the description of the data can be found in Adriani et al. (2011) and García-Comas et al. (2011).

The unidentified emission near $3.28 \mu \mathrm{m}$ has been shown to be much larger than the instrument noise and the errors incurred in its derivation. The VIMS residual spectrum (after subtracting the $\mathrm{CH}_{4}$ contribution) exhibits a clear peak around $3.28 \mu \mathrm{m}$ and just overlaps with the $R$-branch of the strong $\mathrm{CH}_{4} 3.3 \mu \mathrm{m}$ bands (see Figures 1 and 3 in Dinelli et al. 2013 and Figure 4 in this paper). This feature can be seen at altitudes from around $650 \mathrm{~km}$ up to $\sim 1300 \mathrm{~km}$, exhibiting a maximum near $950 \mathrm{~km}$ and a secondary peak at $\sim 1200 \mathrm{~km}$. The estimated errors of the integrated radiance increase significantly as the tangent height decreases, being rather large below around $700 \mathrm{~km}$.

Dinelli et al. (2013) performed a thorough analysis discarding many potential carrier species for the unidentified feature and suggested that it could be due to emission by aromatic compounds. A major problem was how to explain such strong emission from species or particles which should be in much smaller concentrations than methane. PAHs, in addition to having a spectroscopic signature at the wavelength of the "unidentified" emission, are excited by absorption of solar radiation in the UV and very quickly redistribute this energy internally among their different vibrational modes, causing nearand mid-IR emissions (see Figure 1; Tielens 2005). Hence, they are a very likely candidate for explaining the observed emission. We thus analyzed the emission as originating from PAHs. For this, we used the NASA Ames PAH IR Spectroscopic Database (Bauschlicher et al. 2010) and the models developed for the excitation of PAHs by the absorption of solar UV radiation (Boersma et al. 2010, 2011). We should clarify that this database is not restricted to aromatic hydrocarbons, but can also contain other atoms such as, for example, nitrogen. The latter would then follow the nomenclature of HACs. However, since we use the so-called PAH database, we keep this name; even the aromatic species can also contain non-carbon atoms. The next section describes the excitation model for the PAHs and the method used for fitting the spectra and inverting the PAHs abundances. Section 4 describes the major results which are discussed later in Section 5.

\section{EXCITATION OF THE PAHs}

As described above, PAHs can be excited by absorption of solar UV radiation and they very quickly redistribute the energy internally among its different vibrational modes, causing nearand mid-IR emissions, before being re-emitted at the absorption wavelength (see Figure 1; Tielens 2005). We have calculated the efficiencies for the redistribution of the absorbed solar photons into the near-IR vibrational mode, $\bar{\epsilon}_{i}$, by using the "cascade" model as implemented in the AmesPAHdbIDLSuite (Boersma et al. 2010, 2011) and based on the thermal approximation (e.g., Pech et al. 2002). We describe below the methodology used for the analysis of the unidentified emission using these efficiencies.

The limb spectral radiance $L_{v}(h)$ at tangent height $h$ measured by an instrument from a band arising from an excited state (near $3.3 \mu \mathrm{m}$ in this case) of $\mathrm{PAH}_{i}$ with concentration $\left[n_{i}^{*}\right]$ is given by (see Equation (8.16) in López-Puertas \& Taylor 2001)

$$
L_{v}(h)=\int_{\mathrm{FOV}} \int_{\mathrm{LOS}} A_{i}\left[n_{i}^{*}\right] F_{\nu} \varphi_{\nu, i} h v d x d \omega
$$

where $F_{v}$ is the instrument's spectral response, $\varphi_{v, i}$ is the normalized spectral band shape of $\mathrm{PAH}_{i}, A_{i}$ is the Einstein coefficient of the band of $\mathrm{PAH}_{i}$ at work, the integrals are performed over the instrument's field of view (FOV) and along the limb line of sight (LOS), and it is assumed that the band is optically thin. In the derivation of the population $\left[n_{i}^{*}\right]$ of $\mathrm{PAH}_{i}$, 


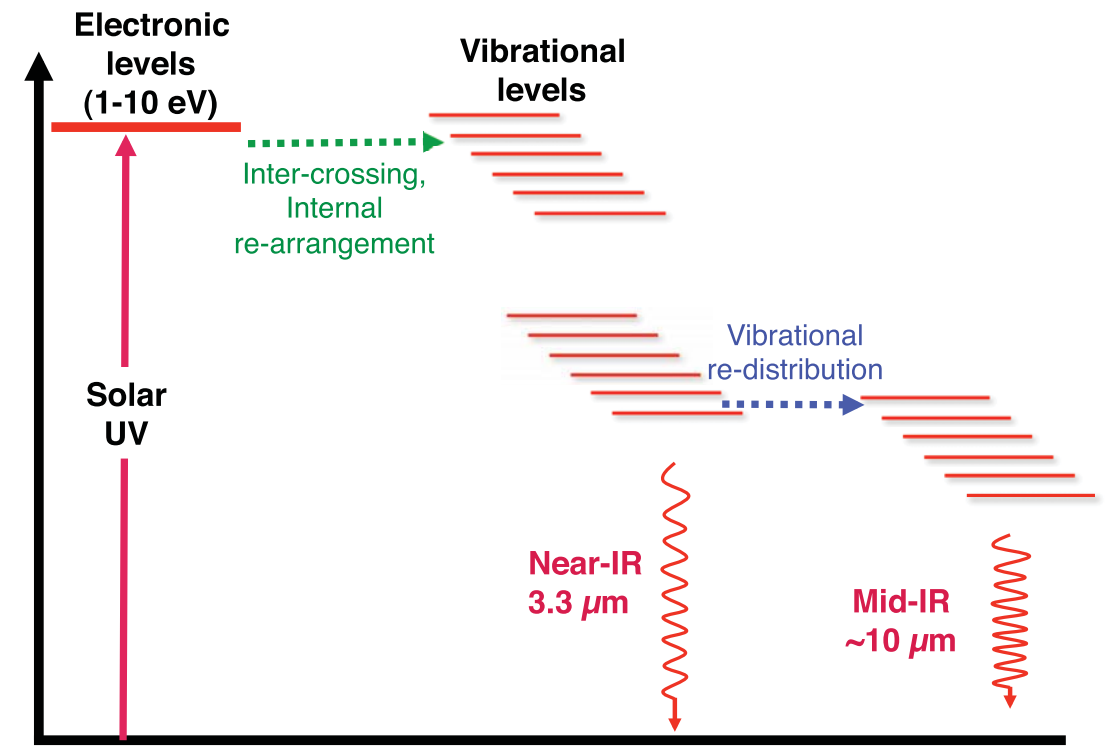

Ground state

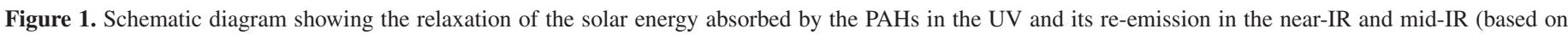
Tielens 2005).

(A color version of this figure is available in the online journal.)

we assumed that: (1) the PAH is excited by absorption of solar photons in the UV-NIR, (2) the excitation and de-excitation by collisions can be neglected given the low pressures of Titan's upper atmosphere, and (3) the only radiative loss of $n_{i}^{*}$ is the spontaneous emission, $A_{i}$, in the near-IR.

Collisional de-activation might be important at the lowest altitudes studied here, i.e., below about 800-900 km but not above. García-Comas et al. (2011) have shown that this is the case for the $\mathrm{CH}_{4} 3.3 \mu \mathrm{m}$ bands. Then, as a first approach, and in the absence of measurements of collisional relaxation, it is plausible to assume that collisions are unimportant as de-excitation of PAHs occurs above around 800-900 km. Actually, the fade down of the emission below about $900 \mathrm{~km}$ (see Figure 2 in Dinelli et al. 2013) suggests that it is caused by the collisional quenching of the UV-excited PAH states rather than by the depletion of their abundances since current models do not predict the decrease of the heavy aromatic species at those altitudes (Vuitton et al. 2008; Lavvas et al. 2011). Hence, as the PAH radiance becomes noisier below about $900 \mathrm{~km}$ (see Figure 2 in Dinelli et al. 2013) and the collisional de-activation might be significant, we limit the derivation of PAH concentrations down to that altitude.

With these assumptions we get, from the statistical equilibrium equation (López-Puertas \& Taylor 2001),

$$
\frac{\left[n_{i}^{*}\right]}{\left[n_{i}\right]}=\frac{J_{\text {sun }, i} \bar{\epsilon}_{i}}{A_{i}},
$$

where $\left[n_{i}\right]$ is the concentration of the $\mathrm{PAH}_{i}$ in the ground state, $J_{\text {sun }, i}$ is the photo-absorption coefficient (in photons $\mathrm{s}^{-1}$ ) of $\mathrm{PAH}_{i}$, and $\bar{\epsilon}_{i}$ is the mean efficiency in terms of the number of $\mathrm{PAH}_{i}$ 's excited in the $3.3 \mu \mathrm{m}$ energy level per solar absorbed photon. $J_{\text {sun }, i}$ is obtained from the integration over wavelength of the solar flux in the UV-NIR range, $\Phi_{\lambda}$, times the $\mathrm{PAH}_{i}$ cross section, $\sigma_{\lambda, i}$, divided by the energy of the solar photon, $h \nu$. Here, we use the cross section of $\mathrm{PAH}_{i}$ in the UV-NIR per carbon atom, $\sigma_{\lambda}$, and assume that its spectral dependence is the same for all PAHs. Then, $\sigma_{\lambda, i}=\sigma_{\lambda} N_{c, i}$, where $N_{c, i}$ is the number of carbons of $\mathrm{PAH}_{i}$. Hence,

$$
J_{\text {sun }, i} \bar{\epsilon}_{i}=N_{c, i} \int_{\lambda, \mathrm{UV}-\mathrm{NIR}} \frac{\Phi_{\lambda} \sigma_{\lambda}}{h v} \epsilon_{i, \lambda} d \lambda=N_{c, i} J_{\text {sun }, c} \bar{\epsilon}_{i}
$$

with

$$
J_{\mathrm{sun}, c}=\int_{\lambda, \mathrm{UV}-\mathrm{NIR}} \frac{\Phi_{\lambda} \sigma_{\lambda}}{h v} d \lambda
$$

and

$$
\bar{\epsilon}_{i}=\frac{\int_{\lambda, \mathrm{UV}-\mathrm{NIR}} \frac{\Phi_{\lambda} \sigma_{\lambda}}{h v} \epsilon_{i, \lambda} d \lambda}{\int_{\lambda, \mathrm{UV}-\mathrm{NIR}} \frac{\Phi_{\lambda} \sigma_{\lambda}}{h v} d \lambda} .
$$

Introducing Equations (2) and (3) into Equation (1) we get

$$
L_{v}(h)=K J_{\text {sun }, c} N_{c, i} \bar{\epsilon}_{i} F_{v} \varphi_{v, i} h v \int_{\text {LOS }}\left[n_{i}\right] d x,
$$

where the constant $K$ is introduced to account for the integration over the instrument's FOV and it is assumed that the integrants barely change within the FOV.

Assuming that the emission comes from several PAHs with different normalized spectral shapes, $\varphi_{v, i}$, and that they are homogeneously distributed along the LOS, the spectral radiance can be written as

$$
L_{v}(h)=K J_{\text {sun }, c}\left(\sum_{i} N_{c, i} \bar{\epsilon}_{i} f_{i} F_{v} \varphi_{v, i} h v\right) \int_{\mathrm{LOS}}[n] d x,
$$

where the ground state concentration of each $\mathrm{PAH}_{i},\left[n_{i}\right]=$ $f_{i}[n], f_{i}$ is the fractional concentration of $\mathrm{PAH}_{i}$ and $[n]$ is the concentration of all PAHs.

In Equation (7) all quantities are known except $f_{i}$ and $[n]$. The photo-absorption coefficients, Equation (4), were calculated using the solar UV flux at the top of Titan's atmosphere 

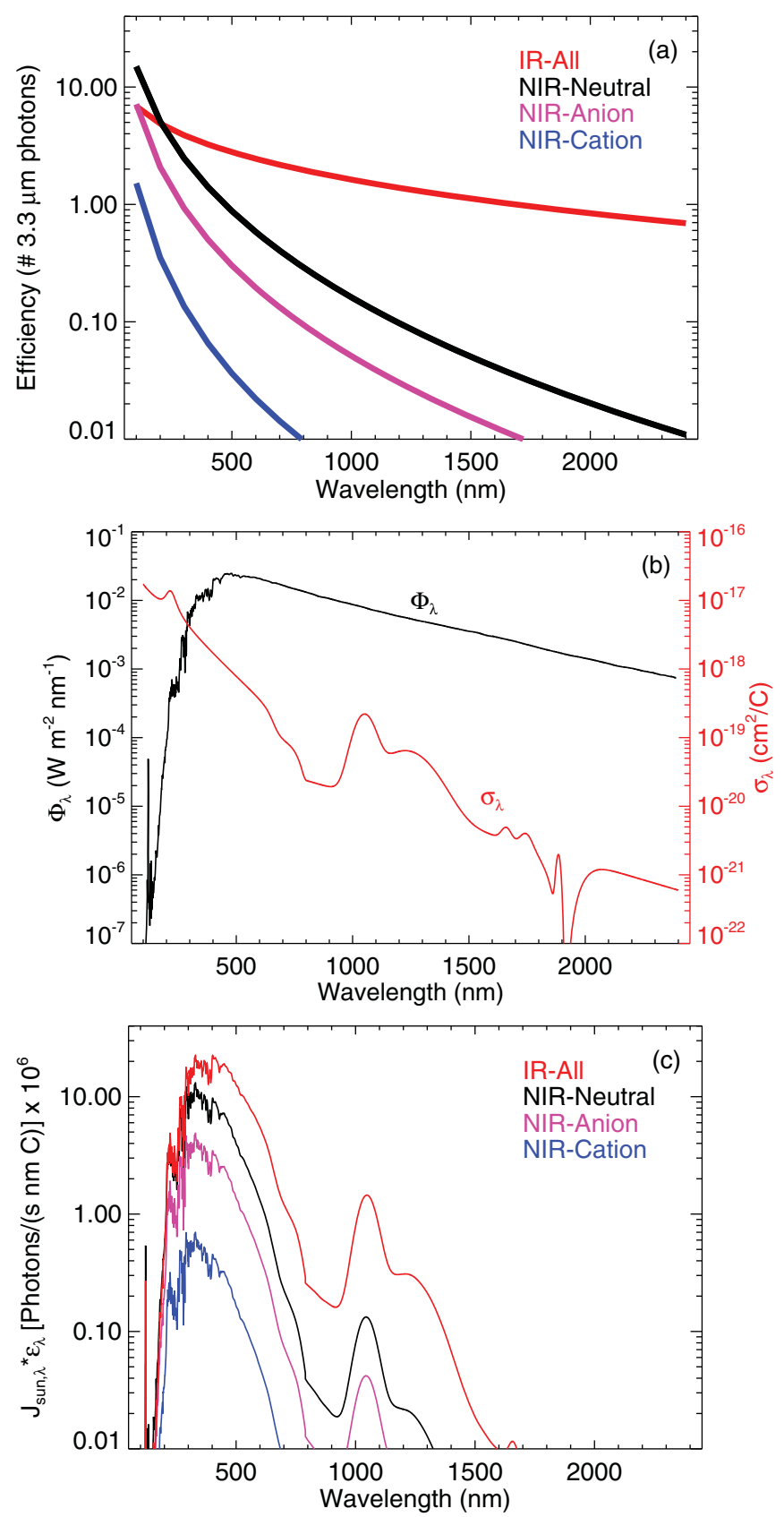

Figure 2. (a) The efficiencies (number of PAHs excited in the $3.3 \mu \mathrm{m}$ energy levels per solar absorbed photon) for the redistribution of the absorbed UV solar photons into the near- and mid-IR vibrational modes, $\epsilon_{i, \lambda}$, calculated with the "cascade" model (Boersma et al. 2010, 2011) for the PAHs considered in this work. The lines show linearly averaged mean efficiencies for: all PAHs in the mid-IR (red); and neutral, anion, and cations in the near-IR (black, magenta, and blue, respectively). (b) Solar flux and the PAHs cross sections. (c) The efficiencies (panel a) multiplied by the solar flux and cross sections (panel b).

(A color version of this figure is available in the online journal.)

taken from the SORCE measurements (Rottman 2005) for the appropriate time of VIMS measurements and scaled to Titan's distance (see Figure 2(b)). Absorption was considered from 0.15 to $2.4 \mu \mathrm{m}$. The $\mathrm{PAH}$ cross sections were taken from Draine \& Li (2007), including the near-IR correction from Mattioda et al. (2005; see Figure 2(b)). The efficiencies for the redistribution of the absorbed solar photons into the near-IR vibrational modes, $\epsilon_{i, \lambda}$, were calculated by using the "cascade" model as implemented in the AmesPAHdbIDLSuite and based

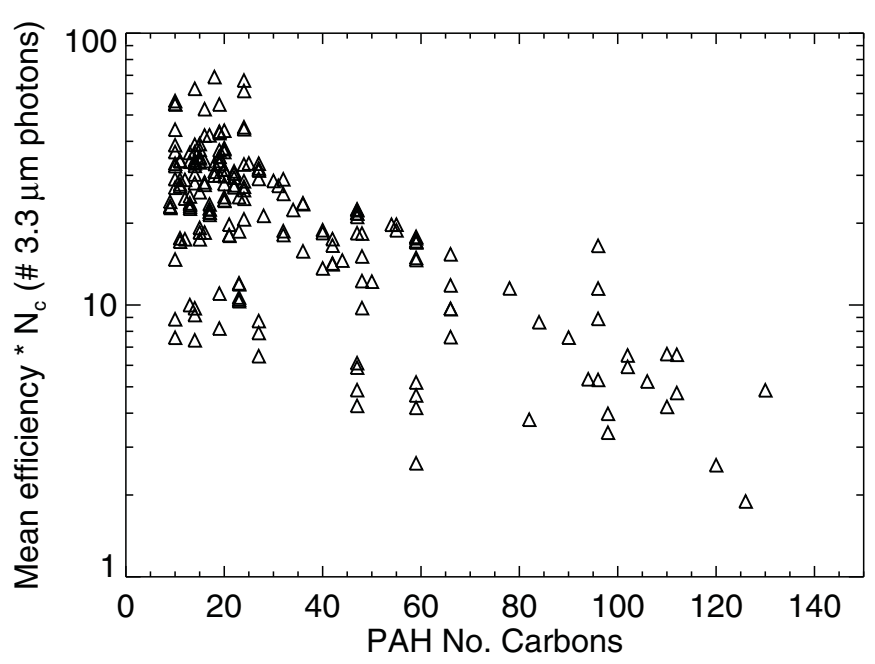

Figure 3. Mean near-IR efficiencies weighted with the solar flux and the cross section, $\overline{\epsilon_{i}}$, according to Equation (5), multiplied by the number of carbons as a function of the number of carbons for the neutral PAHs considered in this work.

on the thermal approximation (e.g., Pech et al. 2002). Figure 2(a) shows the values calculated for the considered PAHs (see the next section) as a function of the energy of the absorbed solar photon. As expected, the energy transferred is larger for the more energetic absorbed photons. Also, they are significantly larger for the neutrals than for the charged PAHs. This is a consequence of their dipole moments: for neutral PAHs the charge accumulates at the edges, where the hydrogen atoms are, inducing a strong dipole moment for the $\mathrm{C}-\mathrm{H}$ modes while, for charged PAHs, the charge is distributed more onto the carbon skeleton, diminishing the dipole moment for the $\mathrm{C}-\mathrm{H}$ modes (Hudgins et al. 1994). Figure 2(c) shows the efficiencies multiplied by the solar flux and the cross section and thus illustrates the wavelengths at which the PAHs are mainly excited by solar radiation. They span, depending on the $\mathrm{PAH}$ and the emitting frequency, from around $200 \mathrm{~nm}$ to $700 \mathrm{~nm}$. Figure 3 represents the mean values, $\bar{\epsilon}_{i}$, weighted with the solar flux and the cross section (see Equation (5)) and multiplied by the number of carbons versus the number of carbons of the PAHs. This illustrates that the PAHs with a number of carbons below about 40 are more efficient in emitting in the near-IR than those with a larger number of carbons.

The VIMS spectral response, $F_{v}$ (see, e.g., Brown et al. 2004; García-Comas et al. 2011), was applied here. The PAH band centers were taken from the NASA Ames PAH IR Spectroscopic Database (Bauschlicher et al. 2010). A Lorentzian band shape with an FWHM of the VIMS spectral resolution $\left(\sim 15 \mathrm{~cm}^{-1}\right)$ was applied to the PAH band strengths (Bauschlicher et al. 2010). Also, a spectral shift of $-15 \mathrm{~cm}^{-1}$ was applied to the PAH spectral bands in order to account for the currently accepted shift between their absorption and emission spectra (see, e.g., Allamandola et al. 1999; Bauschlicher et al. 2010; Boersma et al. 2010, 2011).

The two unknown quantities, $f_{i}$ and $[n]$, are retrieved, respectively, from the shape of the VIMS residual spectra (e.g., those resulting after subtracting the $\mathrm{CH}_{4}$ emission) and from the vertical profile of its integrated radiance. First, we retrieve $f_{i}$, the PAH relative abundances. A non-negative-least-squares (NNLS) approach was used to fit Titan's "unidentified" feature with the emission spectra from the PAHs in the database. More details on the fit procedure are given in Rosenberg et al. (2011) and Boersma et al. (2013). 
Once the fractional $\mathrm{PAH}$ concentrations, $f_{i}$, are derived, the total concentration $[n]$ is retrieved from the geometrical inversion of the limb spectrally integrated radiance, i.e., from the integration over frequency of Equation (7),

$$
L(h)=K J_{\text {sun }, c}\left(\sum_{i} N_{c, i} \bar{\epsilon}_{i} f_{i} \int_{\Delta v} F_{v} \varphi_{v, i} h v d v\right) \int_{\operatorname{LOS}}[n] d x .
$$

\section{SPECTRAL FITTING AND INVERSION OF PAH ABUNDANCES}

The NASA Ames PAH IR Spectroscopic Database at version 1.20 contains 604 PAHs, including neutrals, cations, and anions. Charged particles were not included in the nominal calculations because of their anticipated low abundance in Titan's upper atmosphere (Waite et al. 2007; Vuitton et al. 2008; Krasnopolsky 2009; Tielens 2005), and of their low efficiencies for emitting in the near-IR, about a factor of 10 smaller than those for the neutral PAHs (see Figure 2; Bauschlicher et al. 2010; Boersma et al. 2010, 2011). Also, species containing magnesium, iron, silicon, or oxygen were not considered. This reduced the set of suitable PAHs to 202.

Figures 4(a)-(c) show the fit of the "unidentified" spectral feature for the tangent heights where the spectra have the highest signal-to-noise ratio, i.e., at $1000 \mathrm{~km}, 950 \mathrm{~km}$, and $900 \mathrm{~km}$. The fits were performed considering each PAH spectral band and their near-IR emitting radiances as described above. As it can be seen, the agreement is very good at all altitudes, strongly suggesting that PAHs can be held responsible for the observed "unidentified" emission. Positively charged PAHs emit at slightly shorter wavelengths than their neutral counterparts and negatively charged PAHs emit at slightly longer wavelengths (Bauschlicher et al. 2008). Thus, the underestimation of the radiance at longer wavelengths $(3.32-3.37 \mu \mathrm{m})$ and the weak underestimation (although within the noise) at shorter wavelengths $(3.15-3.20 \mu \mathrm{m})$ are both consistent with the presence of charged PAHs. This underestimation could also be due to the contribution of PAH anharmonic hot bands.

Table 2 lists the 19 most abundant PAHs found in the best fit to the measured spectra, including the three tangent heights shown in Figures 4(a)-(c). We have assessed the mapping of the measurement noise errors onto the fit results by repeating the NNLS fit on the $3.28 \mu \mathrm{m}$ feature by varying its profile within the $1 \sigma$ noise. The results show that essentially the same PAHs appear in all fits, particularly those with larger concentrations (Table 2 lists the mean values and the standard deviations). Only those with minor concentrations (below about 1\%) do not come out in all fits. This suggests that the overall fit is very robust and the most abundant PAHs resulting from the fits and their concentrations are statistically significant.

The total number of carbons in the identified PAHs ranges from 9 to 96 with a concentration-weighted average of 34 carbons. The mean mass is $\sim 430 \mathrm{u}$; the mean area is about $0.53 \mathrm{~nm}^{2}$; and they are formed by $10-11$ rings on average. It is also worth noting that several nitriles (PAHNs) persistently appear in the fit with significant relative concentrations (about one-third of the PAHs contain nitrogen). The mean mass of the identified PAHs is larger than the upper limit of the positive ions measured by CAPS/IBS, but accommodates very well within the ample range of masses of heavy negative ions measured by CAPS/ELS (Waite et al. 2007, 2010; Crary et al. 2009; Coates et al. 2007).
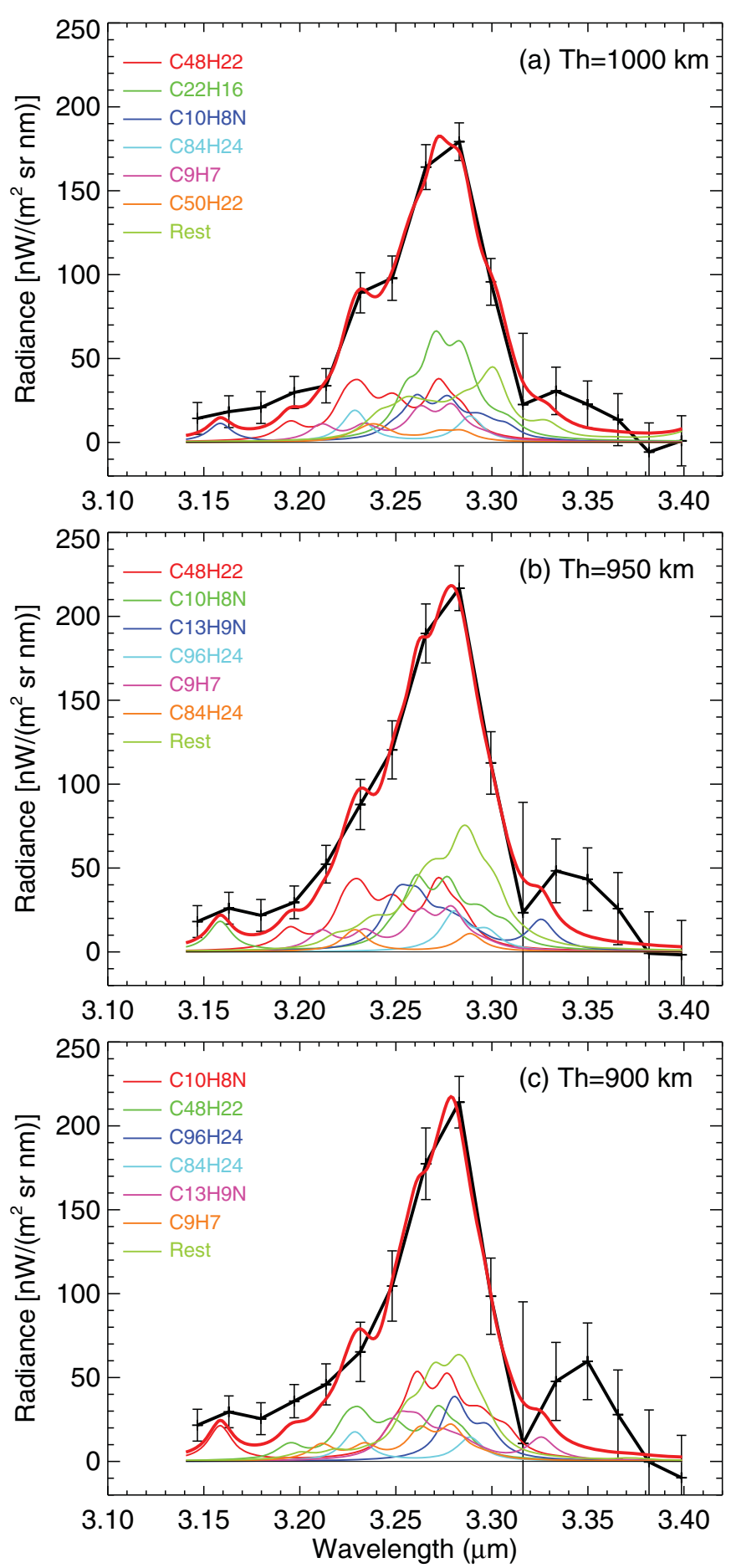

Figure 4. Spectral fit of the VIMS "unidentified" emission spectra with the neutral PAH species in the NASA Ames PAH IR Spectroscopic Database. We used the VIMS measurements listed in Table 1. Measured VIMS spectra and noise errors (black) and the fitted contributing PAHs (individuals in colored thin lines and total in thick red) for tangent heights of $1000 \mathrm{~km}, 950 \mathrm{~km}$, and $900 \mathrm{~km}$. (A color version of this figure is available in the online journal.)

Because the fit is performed with a limited set of PAHs, the results in Table 2 do not necessarily represent the existing PAHs in Titan's upper atmosphere and the presence of other PAHs cannot be ruled out. On the other hand, the lack of measurements at other spectral regions where PAH emits, e.g., in the midIR, restricts absolute identification of individual PAH species. However, since the emission of PAHs in this spectral region is 
Table 2

Relative Concentrations of the Most Abundant PAHs

\begin{tabular}{lclcc}
\hline \hline No. & UID in Database & Chemical Formula & $\begin{array}{c}\text { Concentration } \\
(\%)\end{array}$ & $\begin{array}{c}\text { Precision } \\
(\%)\end{array}$ \\
\hline 1 & & & 22.7 & 11.3 \\
2 & 143 & $\mathrm{C}_{48} \mathrm{H}_{22}$ & 18.6 & 11.5 \\
3 & 527 & $\mathrm{C}_{10} \mathrm{H}_{8} \mathrm{~N}$ & 8.3 & 13.6 \\
4 & 356 & $\mathrm{C}_{22} \mathrm{H}_{16}$ & 8.1 & 6.2 \\
5 & 174 & $\mathrm{C}_{84} \mathrm{H}_{24}$ & 5.4 & 4.7 \\
6 & 276 & $\mathrm{C}_{13} \mathrm{H}_{9} \mathrm{~N}$ & 5.1 & 4.2 \\
7 & 493 & $\mathrm{C}_{9} \mathrm{H}_{7}$ & 4.3 & 4.2 \\
8 & 299 & $\mathrm{C}_{16} \mathrm{H}_{10} \mathrm{~N}_{2}$ & 3.6 & 5.0 \\
9 & 149 & $\mathrm{C}_{50} \mathrm{H}_{22}$ & 3.6 & 6.3 \\
10 & 108 & $\mathrm{C}_{96} \mathrm{H}_{24}$ & 2.4 & 5.2 \\
11 & 473 & $\mathrm{C}_{10} \mathrm{H}_{9} \mathrm{~N}$ & 2.1 & 4.5 \\
12 & 600 & $\mathrm{C}_{66} \mathrm{H}_{20}$ & 1.6 & 3.2 \\
13 & 333 & $\mathrm{C}_{10} \mathrm{H}_{16}$ & 1.3 & 2.4 \\
14 & 385 & $\mathrm{C}_{12} \mathrm{H}_{8}$ & 1.3 & 2.2 \\
15 & 105 & $\mathrm{C}_{42} \mathrm{H}_{18}$ & 1.2 & 2.6 \\
16 & 519 & $\mathrm{C}_{11} \mathrm{H}_{9}$ & 1.1 & 2.4 \\
17 & 267 & $\mathrm{C}_{12} \mathrm{H}_{8} \mathrm{~N}_{2}$ & 0.9 & 1.6 \\
18 & 159 & $\mathrm{C}_{20} \mathrm{H}_{10}$ & 0.8 & 1.1 \\
19 & 339 & $\mathrm{C}_{14} \mathrm{H}_{16}$ & 0.8 & 2.1 \\
\hline & 358 & $\mathrm{C}_{20} \mathrm{H}_{14}$ & & \\
\hline
\end{tabular}

proportional to the number of $\mathrm{C}-\mathrm{H}$ bonds (or rings; Boersma et al. 2010, 2011), the total number of rings obtained here can be considered rather accurate, independent of the derived PAHs. Also, heavier molecules (the heaviest found here is $\mathrm{C}_{96} \mathrm{H}_{24}$, with $1440 \mathrm{u})$ may be present in Titan's upper atmosphere but, if so, the total PAH concentration would then be smaller so the total amount of rings and mass should not change significantly.

By including the excitation model described above and using an Abel inversion, we inverted the limb radiance profile of the "unidentified" emission (see Figure 2 in Dinelli et al. 2013) into a vertical profile of PAH concentrations (Figure 5). The uncertainties introduced by the instrumental errors, mainly the instrument's noise, and by the error in the retrieved $\mathrm{CH}_{4}$ abundance, are also represented in Figure 5. The former is more important at high altitudes while the error of the derived $\mathrm{CH}_{4}$ number density becomes more significant at lower altitudes. The systematic errors (not plotted) are mainly caused by the uncertainty of the efficiency of the energy transfer from the UV to the near-IR and by the inaccuracy in the band strengths of the PAHs. They can be as large as a factor of two (Bauschlicher et al. 2008). We have found that PAHs are present in Titan's upper atmosphere in rather large amounts. We derive a concentration of $(2-3) \times 10^{4}$ particles $\mathrm{cm}^{-3}$ at $\sim 1000 \mathrm{~km}$ which extends to altitudes above $1200 \mathrm{~km}$, where they exhibit a secondary minor peak. Below about $900 \mathrm{~km}$ their near-IR emission declines significantly (see Figure 2 in Dinelli et al. 2013). However, this might be due to the quenching of the excited states becoming significant (as occurs for the $3.3 \mu \mathrm{m}$ levels of $\mathrm{CH}_{4}$; García-Comas et al. 2011), rather than to a depletion of the abundances.

\section{DISCUSSION}

The concentration of PAHs derived here, when expressed in terms of the number of rings, is in reasonable agreement with the number of rings in the form of heavy aromatic species calculated by Vuitton et al. (2008) at an altitude of about $950 \mathrm{~km}$ (see Figure 5). At higher altitudes, the number of rings derived here is, however, significantly larger. The reason for that is unclear although, as pointed by Vuitton et al. (2008), there might be additional pathways to forming aromatics in ion-neutral chemistry to those proposed by these investigators. Indeed, as described

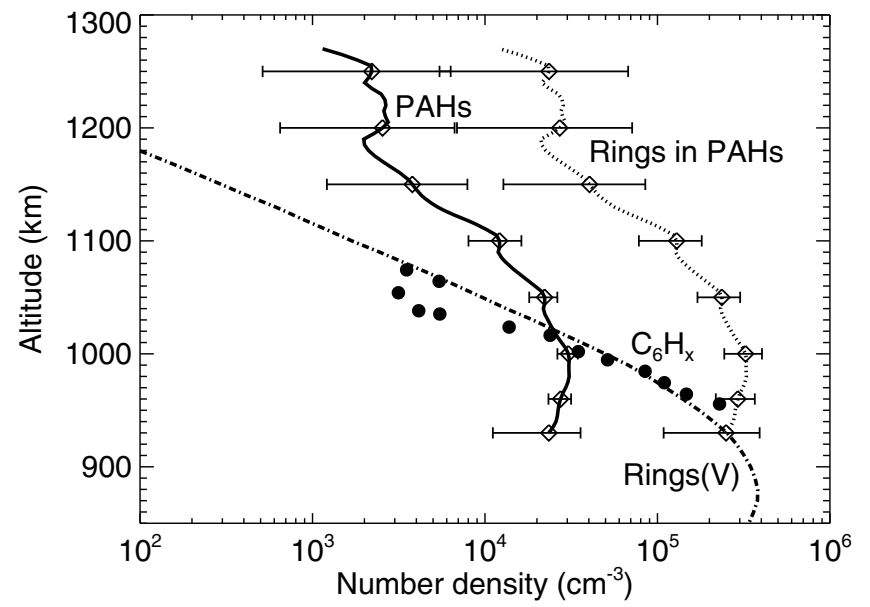

Figure 5. Concentration profile (solid line) of PAHs in Titan's upper atmosphere derived from the VIMS limb profile of the "unidentified" emission in Figure 2 of Dinelli et al. (2013). The uncertainties introduced by the instrumental errors (mainly noise) and by the error in the retrieved $\mathrm{CH}_{4}$ abundance are represented with horizontal bars. The dotted line is the number density of aromatic rings in the PAHs. Its error bars account for the error in the PAH abundances and the standard deviation of the number of rings (see Table 2). The solid dots represent average densities over 12 Titan passes of the combination of benzene and phenyl $\left(\mathrm{C}_{6} \mathrm{H}_{x}\right)$ measured by INMS (see Table 1 in Vuitton et al. 2008). Their errors are estimated to be about $20 \%$. The dot-dashed line, Rings(v), is the ring's number density of heavy aromatic species calculated by Vuitton et al. (2008).

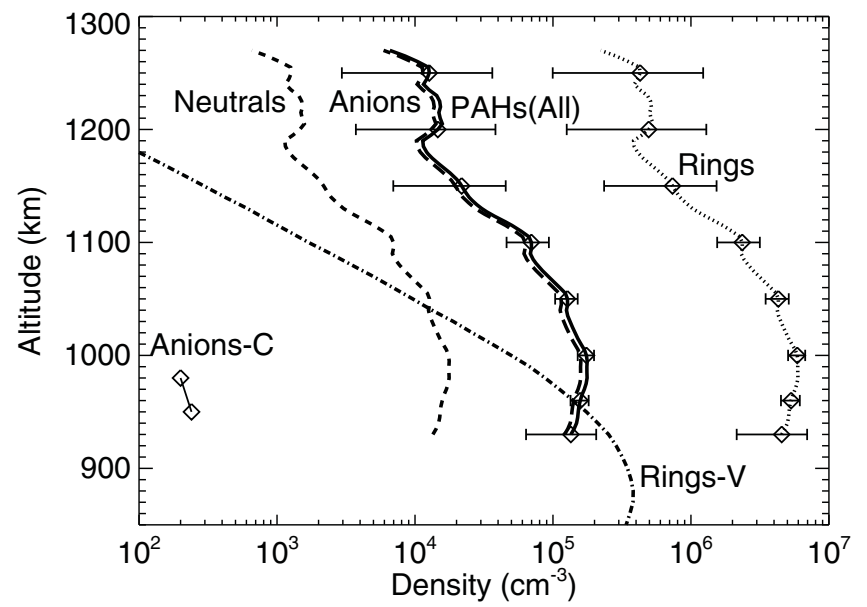

Figure 6. Concentration profiles of PAHs derived by assuming that most of the PAHs are negative ions-neutrals: short-dashed; anions: long-dashed, and total: solid. The dotted line is the number density of aromatic rings in the PAHs. Error bars as in Figure 5 but adapted to this calculation. The dot-dashed line, Rings(v), is the density of heavy aromatic species calculated by Vuitton et al. (2008). The diamonds (Anion-C) represent the concentrations of negative ions measured by CAPS (Coates et al. 2007).

below, the profile shape of the derived PAHs exhibits peaks at similar altitudes than the ion profiles measured by INMS, suggesting that ion-neutral chemistry seems to play a key role. In any case, the density of PAHs derived here does not seem to be unreasonable and, with a density several orders of magnitude smaller than that of $\mathrm{CH}_{4}$, we are able to explain the intense emission observed over the $R$-branch of the $\mathrm{CH}_{4} 3.3 \mu \mathrm{m}$ bands.

We have also tried to fit the "unidentified" emission (1) without imposing any charge restriction on the PAHs and (2) assuming that most of them are negatively charged. The spectral fits obtained are also reasonable but the resulting PAH density is about one order of magnitude larger than when assuming only neutrals (see Figure 6). This is due to the much lesser efficiency of the anions and cations, with respect to the neutrals, 
in re-arranging the UV photons into NIR vibrational energy (see Figures 2(a) and (c)), requiring then a larger PAH abundance to explain the measured radiance. These fits result in unrealistically large densities of PAH ions in Titan's upper atmosphere, several orders of magnitude larger than those measured by CAPS (see Figure 6; Coates et al. 2007). Also, the number of rings obtained is about one order of magnitude larger than those obtained by Vuitton et al. (2008) in their successful explanation of the benzene measurements from INMS.

The current energy relaxation models of PAHs predict that a large part of the absorbed radiation is re-emitted by PAHs in the mid-IR (i.e., at 6.2, 7.7, 8.6, and $11.2 \mu \mathrm{m}$; see Figures 1, and 2(a) and (c)). Therefore, we have analyzed the measurements taken by Cassini/CIRS in the mid-IR spectral region for the sake of finding further confirmation of their presence in Titan's upper atmosphere. The "cascade" model used here (Boersma et al. 2010, 2011) predicts an efficiency for the energy re-emitted in the mid-IR of about a factor of 10 larger than in the near-IR (see Figure 2). Taking the integrated radiance in the near-IR of VIMS, $1 \mathrm{nW}\left(\mathrm{cm}^{2} \mathrm{sr}\right)^{-1}$ (see Figure 2 in Dinelli et al. 2013) and the ratio of the energy available to emit in the mid-IR to the nearIR of $\sim 2$ (derived from Figure 2(a)), we expect a signal of about $2 \mathrm{nW}\left(\mathrm{cm}^{2} \mathrm{sr}\right)^{-1}$ in the mid-IR. This signal, spread over the mid-IR PAH band width of about $200 \mathrm{~cm}^{-1}\left(700-900 \mathrm{~cm}^{-1}\right.$; Bauschlicher et al. 2010; Boersma et al. 2010), results in a spectral radiance of $0.01 \mathrm{nW}\left(\mathrm{cm}^{2} \mathrm{sr} \mathrm{cm}^{-1}\right)^{-1}$. The noise equivalent spectral radiance of CIRS in the $\mathrm{fp} 3$ channel is $10 \mathrm{nW}$ $\left(\mathrm{cm}^{2} \mathrm{sr} \mathrm{cm}^{-1}\right)^{-1}$ (Flasar et al. 2005), which is about a factor of 1000 larger. An average of the available 7500 CIRS fp3 spectra measured in the 800-1100 $\mathrm{km}$ range has shown that the noise of the averaged spectrum is much larger than the expected signal. Thus, unfortunately, CIRS measurements are too noisy for detecting the emission of the PAH concentrations reported here.

Although the PAHs compiled in the NASA Ames PAH IR Spectroscopic Database do not exhibit any other emission feature that falls within the VIMS spectral range $(1-5 \mu \mathrm{m})$, one would expect to have signal from the overtone bands near $1.6 \mu \mathrm{m}$. We have looked at the VIMS spectra and although much noisier than at $3.28 \mu \mathrm{m}$, we have found an emission feature near $1.57 \mu \mathrm{m}$ which could possibly be due to the PAHs. Note that the overtone band of $\mathrm{CH}_{4}$ is significantly displaced to longer wavelengths, i.e., at around $1.6 \mu \mathrm{m}$. On the other hand, that wavelength fits perfectly with the $\mathrm{CO}$ overtone $3 \rightarrow 0$ band. However, this would require a large amount of $\mathrm{CO}$ and should also be observable in the fundamental band near $4.7 \mu \mathrm{m}$, which is not seen above around $600 \mathrm{~km}$. Actually, when reducing the noise by integrating the spectra near $1.57 \mu \mathrm{m}$ and plotting the radiance as a function of tangent height, the shape of the radiance profile resembles very much that of the emission near $3.28 \mu \mathrm{m}$ of Figure 2 in Dinelli et al. (2013), with maxima at exactly the same tangent heights of 950 and $1200 \mathrm{~km}$. This suggests that that emission could be due to PAHs as well. However, since the signal near $1.57 \mu \mathrm{m}$ is much weaker and the spectroscopic data and excitation of the PAHs in this spectral region are not known, we have not used this emission for retrieving or constraining the PAH concentrations.

The vertical distribution of the PAHs coincides with the region where the ion concentrations measured by INMS and CAPS/IBS have their maximum values (Vuitton et al. 2008; Waite et al. 2010). Also, the measurements of the positive ions taken by INMS and CAPS/IBS exhibit a peak near $1250 \mathrm{~km}$ (Vuitton et al. 2008; Coates et al. 2007, 2010; Waite et al. 2010) that coincides with the secondary peak of the PAHs found here. This then suggests that ions play an important role in the formation of heavy PAHs.

The measured PAH concentrations are also consistent with the concentrations of positive and negative ions measured by INMS and CAPS. Krasnopolsky (2009) found, starting from the heavy negative ions measured by CAPS/ELS and typical electron densities of $10^{3} \mathrm{~cm}^{-3}$, and assuming that the positive and negative ions are formed from PAHs by a balance between electron attachment and photo-detachment, a concentration of heavy PAHs of $1.5 \times 10^{4} \mathrm{~cm}^{-3}$ at $\sim 950 \mathrm{~km}$. This value is in excellent agreement with the concentration of $\sim 2 \times 10^{4} \mathrm{~cm}^{-3}$ found here (Figure 5).

Evidence of heavy aerosol particles in Titan's upper atmosphere has been reported from Cassini/UVIS (Liang et al. 2007). These authors derived concentrations of the spherical particles of $\sim 60$ particles $\mathrm{cm}^{-3}$ at $1000 \mathrm{~km}$. The extinctions of that concentration at 163.1 and $238.4 \mathrm{~nm}$ are $1.2 \times 10^{-11}$ and $7.2 \times 10^{-11} \mathrm{~cm}^{-1}$, respectively. Calculations of the extinctions produced by the PAHs derived here, taking into account their cross sections (Figure 2(b)), results in values of $1.12 \times 10^{-11}$ and $1.04 \times 10^{-11} \mathrm{~cm}^{-1}$, which are very similar to those of the particles measured by Liang et al. (2007). Hence, the particles observed by UVIS at high altitudes could then very likely be the PAHs detected here.

Therefore, there are several pieces of evidence suggesting that the PAHs reported here are the neutral counterparts of the heavy negative ions measured by CAPS/ELS and the aerosols measured by UVIS and, consequently, that they play a key role in the formation of the major haze layer of Titan's stratosphere.

\section{SUMMARY AND CONCLUSIONS}

In this paper, we have analyzed the strong unidentified emission near $3.28 \mu \mathrm{m}$ in Titan's upper daytime atmosphere recently reported by Dinelli et al. (2013). The emission has been analyzed by using the NASA Ames PAH IR Spectroscopic Database and the AmesPAHdbIDLSuite set of IDL tools, appropriately modified. Based on excitation models for PAHs, where they are excited by absorption of solar UV radiation and that energy quickly re-arranged internally into the nearIR vibrational modes, we have been able to explain with great consistency the observed spectral feature and derive the vertical distribution of the PAHs abundance in Titan's upper atmosphere.

We have found that PAHs are present in large concentrations in the upper atmosphere, with a peak concentration of about $(2-3) \times 10^{4}$ particles $\mathrm{cm}^{-3}$ and extending up to about $1250 \mathrm{~km}$, where they present a secondary minor peak. The major source of error in these concentrations is the uncertainties in the excitation modeling of the PAHs as well on their band strengths, which can be as large as a factor of two. The identified PAHs have 9-96 carbons, with a concentration-weighted average of 34. The mean mass is $\sim 430 \mathrm{u}$; the mean area is about $0.53 \mathrm{~nm}^{2}$; they are formed by 10-11 rings on average, and about one-third of them contain nitrogen. Although the PAHs distribution derived here might not be exactly those present in Titan's upper atmosphere, the total number of aromatic rings and the total mass derived here can be considered as rather accurate.

The good agreement between the PAH concentration derived here and the estimation of complex molecules abundance by Krasnopolsky (2009), based on the concentrations of heavy negative ions measured by CAPS/ELS and typical electron densities, suggests that they are the neutral counterpart of those heavy positive and negative ions. Also, the good coincidence 
in altitude of the secondary peak of the PAHs and the peak of the positive ions measured by INMS and IBS (Waite et al. 2010) near $1250 \mathrm{~km}$ suggests that ions play a crucial role in the formation of PAHs. Therefore, we think that this finding is a major step forward supporting, as suggested earlier (Waite et al. 2007; Vuitton et al. 2008; Krasnopolsky 2009; Lavvas et al. 2011), that the source of the main haze layer of Titan is located in the upper atmosphere.

The PAH excitation models also predict that a significant fraction of the absorbed UV solar energy goes into the midIR vibrational modes. Unfortunately, the predicted signal to be emitted in the mid-IR is too weak to be detected by the CIRS instrument. As for the future, more sensitive IR instruments as well as near-IR instrument with better spectral resolution than VIMS would be very useful to further discern the composition of the PAHs. In addition, modeling efforts to improve the accuracy of the energy transfer from UV into near- and mid-IR, as well as the PAH $3.28 \mu \mathrm{m}$ band strengths, also including their hot and anharmonic effects on band profile shape, are of very high interest.

Special thanks are given to the Cassini/VIMS team for planning the VIMS observations and to Nick Teanby for looking at and providing the CIRS high-altitude spectra. The activities of the Italian authors have been supported by the Italian Space Agency under the grant ASI I/015/09. The IAA team was supported by the Spanish MINECO under grant AYA2011-23552, the CONSOLIDER program CSD2009-00038, and EC FEDER funds. M.G.C. is also financially supported by MINECO under its "Ramón y Cajal" subprogram. C.B.'s work was supported by an appointment to the NASA Postdoctoral Program at the Ames Research Center, administered by Oak Ridge Associated Universities through a contract with NASA.

Facilities: Cassini (CIRS, VIMS)

\section{REFERENCES}

Adriani, A., Dinelli, B., López-Puertas, M., et al. 2011, Icar, 214, 584 Allamandola, L. J., Hudgins, D. M., \& Sandford, S. A. 1999, ApJL, 511, L115
Atreya, S. K. 2007, Sci, 316, 843

Bauschlicher, C. W., Boersma, C., Ricca, A., et al. 2010, ApJS, 189,341

Bauschlicher, C. W., Peeters, E., \& Allamandola, L. J. 2008, ApJ, 678,316

Boersma, C., Bauschlicher, C. W., Ricca, A., et al. 2011, ApJ, 729, 64

Boersma, C., Bauschlicher, C. W., Jr., Allamandola, L. J., et al. 2010, A\&A, 511, A32

Boersma, C., Bregman, J., \& Allamandola, L. J. 2013, ApJ, 769, 117

Brown, R. H., Baines, K. H., Bellucci, G., et al. 2004, SSRv, 115, 111

Coates, A., Crary, F., Lewis, G., et al. 2007, GeoRL, 34, L22103

Coates, A. J., Wellbrock, A., Lewis, G. R., et al. 2010, P\&SS, 57, 1866

Coustenis, A., Salama, A., Schulz, B., et al. 2003, Icar, 161, 383

Crary, F. J., Magee, B. A., Mandt, K., et al. 2009, P\&SS, 57, 1847

Dinelli, B. M., López-Puertas, M., Adriani, A., et al. 2013, GeoRL, in press

Draine, B. T., \& Li, A. 2007, ApJ, 657, 810

Flasar, F., Achterberg, R., Conrath, B., et al. 2005, Sci, 308, 975

García-Comas, M., López-Puertas, M., Funke, B., Molina, A., \& Coradini, A. 2011, Icar, 214, 571

Hudgins, D. M., Sandford, S. A., \& Allamandola, L. J. 1994, JPhCh, 98, 4243

Krasnopolsky, V. A. 2009, Icar, 201, 226

Lavvas, P., Sander, M., Kraft, M., \& Imanaka, H. 2011, ApJ, 728, 80

Lebonnois, S., Bakes, E., \& McKay, C. P. 2002, Icar, 159, 505

Liang, M.-C., Yung, Y. L., \& Shemansky, D. E. 2007, ApJ, 661, L199

López-Puertas, M., \& Taylor, F. W. 2001, Non-LTE Radiative Transfer in the Atmosphere (Singapore: World Scientific)

Mattioda, A. L., Allamandola, L. J., \& Hudgins, D. M. 2005, ApJ, 629, 1183

Pech, C., Joblin, C., \& Boissel, P. 2002, A\&A, 388, 639

Ricketts, C. L., Schröder, D., Alcaraz, C., \& Roithová, J. 2008, CEJ, 14, 4779

Rosenberg, M. J. F., Berné, O., Boersma, C., Allamandola, L. J., \& Tielens, A. G. G. M. 2011, A\&A, 532, A128

Rottman, G. 2005, SoPh, 230, 7

Sagan, C., Khare, B. N., \& Lewis, J. S. 1984, Organic Matter in the Saturn System (Tucson, AZ: Univ. Arizona Press), 788

Sagan, C., Khare, B. N., Thompson, W. R., et al. 1993, ApJ, 414, 399

Smith, B. A., Soderblom, L., Beebe, R., et al. 1981, Sci, 212, 163

Tielens, A. G. G. M. 2005, The Physics and Chemistry of the Interstellar Medium (Cambridge: Cambridge Univ. Press)

Vuitton, V., Yelle, R. V., \& Cui, J. 2008, JGR, 113, E05007

Waite, J. H., Young, D. T., Cravens, T. E., et al. 2007, Sci, 316, 870

Waite, J. H., Young, D. T., Westlake, J. H., et al. 2010, High-altitude Production of Titan's Aerosols (Dordrecht: Springer), 201

Wilson, E. H., \& Atreya, S. K. 2003, P\&SS, 51, 1017

Wilson, E. H., Atreya, S. K., \& Coustenis, A. 2003, JGR, 108, 5014

Yung, Y. L., Allen, M., \& Pinto, J. P. 1984, ApJS, 55, 465 